Results 64 patients underwent EMR for HGD (20) or early oesophageal cancer (44) in BE. 149 EMR's were performed. 48 patients were male (75\%), mean age was 70.6years (range 30-87years). 20 patients $(31 \%)$ with either submucosal or deeper infiltration or poor differentiation in the EMR specimen underwent surgery/chemotherapy or were conservatively managed depending on patient's fitness, comorbidities and choice. Mean follow-up of the remaining 44 patients undergoing endoscopic therapy was 22.7 month (range 1-55 months). Remission of dysplasia/neoplasia was achieved in $97.9 \%$. Stepwise EMR during follow up resulted in complete ablation of BE in 18 patients (40\%) in a mean of 4 sessions. All patients with complete endoscopic Barrett's ablation had an initial maximal Barrett lengths $<3 \mathrm{~cm} .7$ patients with long Barrett's $(>5 \mathrm{~cm}$ ) received RFA and 7 others are awaiting the procedure. The overall complication rate was low (6.7\%) including a stricture after RFA $(0.7 \%), 8$ procedural $(5.3 \%$, none $\mathrm{Hb}$ relevant) and a delayed bleeding $(0.7 \%)$ but no perforations. All complications were managed endoscopically.

Conclusion Oesophageal EMR is a safe procedure which can achieve remission of neoplasia. In short segment Barrett's oesophagus it can also result in complete ablation of BE. Stepwise endoscopic resection for ablation has a low complication rate when performed in 3 monthly follow-up intervals.

Disclosure of Interest None Declared

\section{PTU-050 EFFICACY OF ENDOSCOPICALLY APPLIED 5\% INTRA- RECTAL FORMALIN TO TREAT RADIATION-INDUCED RECTAL BLEEDING}

doi:10.1136/gutjnl-2013-304907.142

1,"H Jolly, ${ }^{1}$ A C Muls, 'H J Andreyev. 'Royal Marsden Hospital, London, UK

Introduction Up to $50 \%$ of patients develop rectal bleeding after pelvic radiotherapy. Radiation-induced bleeding may be trivial and after appropriate endoscopic assessment, patients can be reassured and do not need treatment. Others develop troublesome bleeding affecting quality of life. 1-5\% become transfusion dependent. The optimal treatment of radiation-induced bleeding and safety of available treatment modalities is controversial.

Methods A service evaluation was performed to assess outcomes of patients treated with intra-rectal formalin for radiation-induced rectal bleeding. Patients were offered formalin treatment if bleeding affected quality of life, they had no evidence of rectal ulceration and bowel function had been otherwise optimised. 5\% formalin was delivered to the rectum using a gastroscope after full bowel preparation with the unsedated patient lying prone. Wet gauze was firmly applied to the perineum to prevent formalin leakage. $30-35 \mathrm{mls}$ formalin, sufficient to cover the telangiectasia, was instilled. The gastroscope was not removed. After 3 minutes, the formalin was washed out with water. If required, treatments were repeated 6-8 weeks later. Patients completed a questionnaire before each treatment and at follow-up. They were also asked to indicate on a $10 \mathrm{~cm}$ visual analogue scale (VAS) the degree of nuisance currently caused by bleeding.

Results 27 men, median age 73 (range 56-81) were treated between 2008 and 2012. All received radiotherapy for prostate cancer in the previous 4 years. $41 \%(n=11)$ had required $\geq 1$ blood transfusions, $48 \%(n=13)$ iron supplementation. $52 \%(n=14)$ regularly soiled their clothes with blood. $22 \%(n=6)$ had not responded to previous argon plasma coagulation. Median follow-up was 28 months (range 2-48).

$67 \%(\mathrm{n}=18)$ reported improvement; reduction in frequency $(52 \%, n=14)$, and/or amount $(44 \%, n=12)$ of bleeding. Patients required 1-4 treatments (median 2) to achieve adequate improvement. Median baseline VAS score was $6.7 \mathrm{~cm}$ (range 4.3-9.9). After one treatment, VAS scores improved by a median of $2.5 \mathrm{~cm}$ (range
0.6-5.0), after 2 treatments by $3 \mathrm{~cm}$ from baseline (range $0.7-6.1 \mathrm{~cm}$ ) and at follow up by $3.8 \mathrm{~cm}$ (range $0-8.7$ ). Two patients experienced serious complications. One who had chronic lymphocytic leukaemia developed septicaemia and pneumonia the following day. The organism responsible was not a bowel organism. The other developed an anterior rectal wall fistula in an area of intense radiation change requiring surgery 18 months later. Neither complication was considered to be caused by the formalin.

Conclusion $67 \%$ patients with radiation-induced rectal bleeding experience a satisfactory reduction in bleeding following treatment with intra-rectal formalin. Further studies comparing with other treatment modalities are required.

Disclosure of Interest None Declared

\section{Gl physiology}

\section{PTU-051 POST-PARTUM DEFECATORY DYSFUNCTION: A COMPLEX PROBLEM INVOLVING CO-EXISTING FAECAL INCONTINENCE AND RECTAL EVACUATORY DISORDER}

doi:10.1136/gutjnl-2013-304907.143

1. ${ }^{*}$ E Horrocks, ${ }^{1 P}$ Jordan, ${ }^{2} \mathrm{R}$ Burgell, ${ }^{2} \mathrm{M}$ Scott, ${ }^{1} \mathrm{C}$ Knowles. ${ }^{1}$ National Centre for Bowel Research and Surgical Innovation; ' Wingate Institute of Neurogastroenterology, Queen Mary University London, London, UK

Introduction Faecal incontinence and urgency are well-recognised sequelae of obstetric injury, however there is a paucity of literature describing the co-existence of symptoms of rectal evacuatory dysfunction (RED) and faecal incontinence (FI). This study aims to analyse and quantify these symptoms in patients referred for anorectal investigations following obstetric injury.

Methods Consecutive patients undergoing specialist investigation at a tertiary referral unit following obstetric injury between $1^{\text {st }}$ July 2010 and $31^{\text {st }}$ July 2012 were identified. Symptoms at presentation were ascertained from the history. Patients routinely complete a Cleveland Clinic Constipation Score (CCCS) and Vaizey Incontinence score (VIS) prior to investigation. Symptoms volunteered by the patient were correlated with formal scoring systems to verify accuracy of symptom reporting and to study the co-existence of FI and RED.

Results One hundred and sixty five women [median age 34 (range 19-55)] were included. Median parity was 2 [range 1-8]. Fourteen women $(9.7 \%)$ had a 4 th degree tear and 68 women $(41 \%)$ a $3^{\text {rd }}$ degree tear [106 (64\%) occurring from the first vaginal delivery]. Three women requiring de-functioning stoma were excluded from further analysis.

FI was volunteered in 87 women (54\%), [59 urge FI (36\%), 57 passive FI (35\%) and 28 mixed FI (17\%)]. Ninety women $(56 \%)$ volunteered symptoms of RED. Co-existent symptoms of RED and FI were present in 58 women (36\%).

VIS and CCCS were available for 79 patients. Median VIS was 8 (IOR 4-13), and was significantly higher in those reporting FI symptoms [median score 11 (IOR 8-15)] than those not [median score 4 (IOR 0-8)] ( $p<0.0001$ Mann Whitney U Test). Median CCCS was 8 (IOR 4-14), which was significantly higher in those reporting RED symptoms [median score 13 (IOR 11-17)] than those not [median score 5 (IOR 3-8)] ( $<0.0001$ Mann Whitney U Test). Fifty four patients (68\%) had significant FI based on VIS (score > 5) and 39 patients (49\%) had significant RED based on CCCS (score $>8$ ). Thirty patients $(38 \%)$ had scores compatible with significant co-existent FI and RED.

Conclusion These results demonstrate symptoms of FI and RED often co-exist following obstetric injury regardless of method used to ascertain symptoms. A multi-modality treatment approach addressing overlapping pathophysiologies should be considered in these patients.

Disclosure of Interest None Declared 